\title{
Erratum to: The Backward Problem for Ginzburg-Landau-Type Equation
}

\author{
Dang Duc Trong1 • Bui Thanh Duy ${ }^{2}$. \\ Nguyen Dang Minh ${ }^{3}$
}

Received: 3 December 2013 / Revised: 28 July 2014 / Accepted: 4 August 2014 /

Published online: 18 September 2015

(C) Institute of Mathematics, Vietnam Academy of Science and Technology (VAST) and Springer

Science+Business Media Singapore 2015

\section{Erratum to: Acta Math Vietnam \\ DOI 10.1007/s40306-015-0135-y}

We regret the original published paper contained a typo within article title.

The original published title;

\section{The Backward Problem for Ginzurg-Landau-Type Equation}

The title replacing the original published and the only one appropriate;

The Backward Problem for Ginzburg-Landau-Type Equation

The online version of the original article can be found under doi: 10.1007/s40306-015-0135-y.

Bui Thanh Duy

duybui55@gmail.com

Dang Duc Trong

ddtrong@hcmus.edu.vn

Nguyen Dang Minh

ndminhsv1986@gmail.com

1 Faculty of Mathematics and Computer Science, University of Science, Ho Chi Minh City National University, No. 227 Nguyen Van Cu Street, Ward 4, District 5, Ho Chi Minh City, Vietnam

2 Faculty of Fundamental Science, Ho Chi Minh City Architecture University, No. 196 Pasteur Street, Ward 6, District 3, Ho Chi Minh City, Vietnam

3 Center for Mathematical Science, University of Science, Ho Chi Minh City National University, No. 227 Nguyen Van Cu Street, Ward 4, District 5, Ho Chi Minh City, Vietnam 